\title{
Inhaled antibiotics: dry or wet?
}

\author{
Harm A.W.M. Tiddens ${ }^{1,2}$, Aukje C. Bos ${ }^{1,2}$, Johan W. Mouton ${ }^{3}$, \\ Sunalene Devadason ${ }^{4}$ and Hettie M. Janssens ${ }^{1}$
}

\section{Affiliations:}

'Erasmus MC-Sophia Children's Hospital, Dept of Pediatric Pulmonology and Allergology, Rotterdam, The Netherlands.

${ }^{2}$ Erasmus MC, Dept of Radiology, Rotterdam, The Netherlands.

${ }^{3}$ Erasmus MC, Dept of Medical Microbiology and Infectious Diseases, Rotterdam, The Netherlands.

${ }^{4}$ School of Paediatrics and Child Health, University of Western Australia, Perth, Australia.

\section{Correspondence:}

Harm A.W.M. Tiddens, Erasmus MC-Sophia, Dept of Pediatric Pulmonology and Allergology, Wytemaweg 80 , 3015 CN, Rotterdam, The Netherlands.

E-mail: h.tiddensderasmusmc.nl

ABSTRACT Dry powder inhalers (DPIs) delivering antibiotics for the suppressive treatment of Pseudomonas aeruginosa in cystic fibrosis patients were developed recently and are now increasingly replacing time-consuming nebuliser therapy. Noninferiority studies have shown that the efficacy of inhaled tobramycin delivered by DPI was similar to that of wet nebulisation. However, there are many differences between inhaled antibiotic therapy delivered by DPI and by nebuliser. The question is whether and to what extent inhalation technique and other patient-related factors affect the efficacy of antibiotics delivered by DPI compared with nebulisers. Health professionals should be aware of the differences between dry and wet aerosols, and of patient-related factors that can influence efficacy, in order to personalise treatment, to give appropriate instructions to patients and to better understand the response to the treatment after switching.

In this review, key issues of aerosol therapy are discussed in relation to inhaled antibiotic therapy with the aim of optimising the use of both nebulised and DPI antibiotics by patients. An example of these issues is the relationship between airway generation, structural lung changes and local concentrations of the inhaled antibiotics. The pros and cons of dry and wet modes of delivery for inhaled antibiotics are discussed.

@ERSpublications

Concentrations of inhaled antibiotics depend on airway generation, mode of delivery, disease severity and competence http://ow.ly/AJLs9

Received: May 162014 | Accepted after revision: Aug 112014

Conflict of interest: Disclosures can be found alongside the online version of this article at erj.ersjournals.com 


\section{Introduction}

Cystic fibrosis (CF) lung disease results in abnormal secretions in the lung that foster infection and inflammation, even early in life $[1,2]$. The vicious cycle of infection, inflammation and thick pulmonary secretions leads to early structural lung damage and to abnormal pulmonary function tests. In addition to bronchiectasis, small airways play an important role in early CF lung disease [3]. In advanced lung disease, the geometric changes related to small airway disease are significantly more severe relative to large airway changes. Progressive bronchiectasis and small airway disease eventually lead to end-stage lung disease [4]. It has long been recognised that pulmonary infection, particularly by Pseudomonas aeruginosa, is associated with progressive structural lung damage [2]. For this reason, after showing that eradication therapy using nebulised antibiotics was effective in preventing chronic infection by $P$. aeruginosa [5-7], this has become standard of treatment $[8,9]$. For those patients who develop chronic $P$. aeruginosa infection, maintenance treatment using nebulised antibiotics has become the standard of treatment to suppress this microorganism chronically [8-11].

Nebulised antibiotics against $P$. aeruginosa were developed as an alternative for intravenous therapy to deliver high concentrations of the antimicrobial agent directly to the site of infection, with the dual aims of improving efficacy and reducing toxicity. Nebulised tobramycin, colistin and aztreonam lysine are the most commonly used nebulised antibiotics for this indication. Of these inhaled antibiotics, tobramycin inhalation solution (TIS) has been studied most extensively. TIS should be used in a " 1 month on and 1 month off" treatment cycle. Maintenance treatment with TIS has been shown to reduce exacerbations, improve lung function and improve quality of life [10, 12]. In addition, early treatment using inhaled nebulised tobramycin against $P$. aeruginosa given at the time of first isolation prevents chronic infection in about twothirds of patients $[7,13,14]$. Similar efficacy was recently observed in a study with nebulised aztreonam lysine [15]. Furthermore, treatment with nebulised aztreonam lysine has been shown to be effective in delaying the need for inhaled or intravenous anti-P. aeruginosa antibiotics for pulmonary exacerbations in CF patients, and in an improved quality of life [16]. Nebulised colistin is generally used as continuous treatment to suppress $P$. aeruginosa growth $[17,18]$.

Until recently, antibiotic maintenance treatment for chronic $P$. aeruginosa infection could only be delivered by nebulisers. Unfortunately, nebulisers have many disadvantages such as the need for rigorous cleaning after each use to reduce the risk for contamination, they are relatively bulky to carry around, and nebulisation time, particularly for older systems, can be lengthy. More recently, a tobramycin inhalation powder (TIP) inhaler was developed as a more patient-friendly alternative to TIS [19]. In general, dry powder inhalers (DPIs) allow fast delivery, are more portable, require minimal cleaning and are disposable, reducing the risk of contamination. Similarly, a DPI for colistin (Colobreathe; Forest Laboratories Inc., New York, NY, USA) has also recently been developed $[18,20]$. Other antibiotics that are in development as DPI formulations are ciprofloxacin, levofloxacin, vancomycin and clarithromycin [21-25]. Regulatory studies of TIP and Colobreathe have shown noninferiority relative to the nebulised solutions. However, even though these regulatory studies of TIP and Colobreathe showed equal efficacy of the DPI compared with the nebulised inhaled formulation, one should take into consideration that there are many patient- and devicerelated differences between the two inhalation modalities that might affect the efficacy of treatment. It is unlikely that the efficacy of antibiotics delivered by DPI is equivalent to that of wet nebulisation for all patients.

Health professionals prescribing inhaled antibiotics for the treatment of chronic P. aeruginosa in CF or in other patients groups such as non-CF bronchiectasis [26, 27] should be well aware of differences in administration between DPIs and nebulisers to allow them to identify patients who might benefit from switching from nebuliser to DPI treatment, and to enable them to give appropriate instructions to patients. The aim of this review is to discuss key issues related to inhaled antibiotic therapy to optimise the effectiveness of both nebulised and DPI antibiotics.

\section{Aerosol particles and deposition}

For efficient aerosol treatment of both central and small airways, it is important to consider a number of factors that determine whether a sufficient fraction of the inhaled particles are able to bypass the upper airways and to be deposited onto the target area, namely the large and small airways. These factors can be divided into particle-related factors and patient-related factors.

The aerodynamic behaviour of a particle depends on shape, size and density of the particles. The size distribution of an aerosol is usually described as the mass median aerodynamic diameter (MMAD), which refers to the droplet diameter above and below which $50 \%$ of the mass of drug is contained. In general, aerosol particles smaller than $5 \mu \mathrm{m}$ are thought to be respirable. However, particles with a MMAD between 2 and $5 \mu \mathrm{m}$ have a lower probability of bypassing the upper airways and of being transported to and deposited in the small airways relative to $1-2-\mu \mathrm{m}$ particles (fig. 1). Unfortunately, small particles carry little 


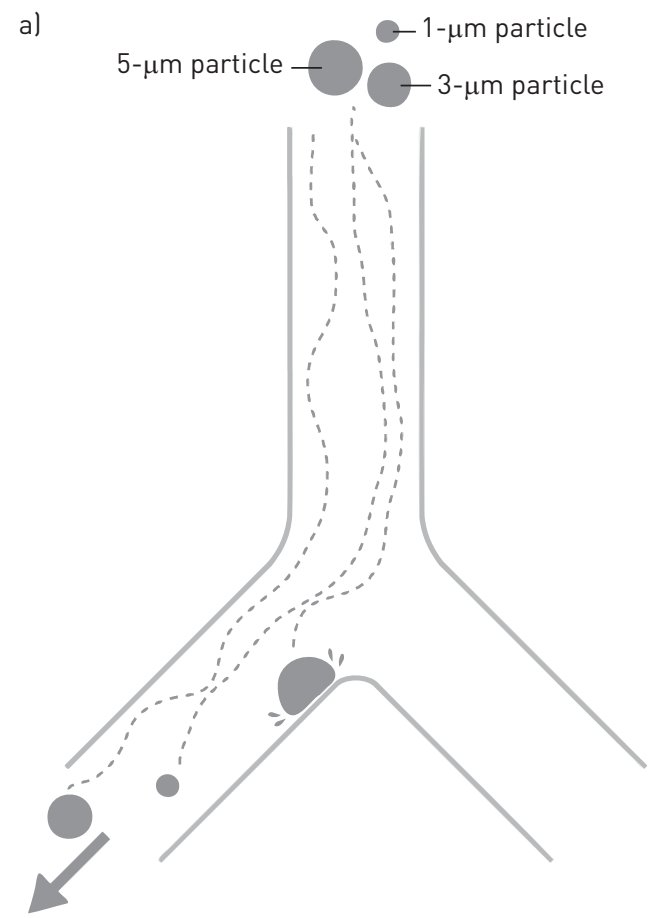

b)

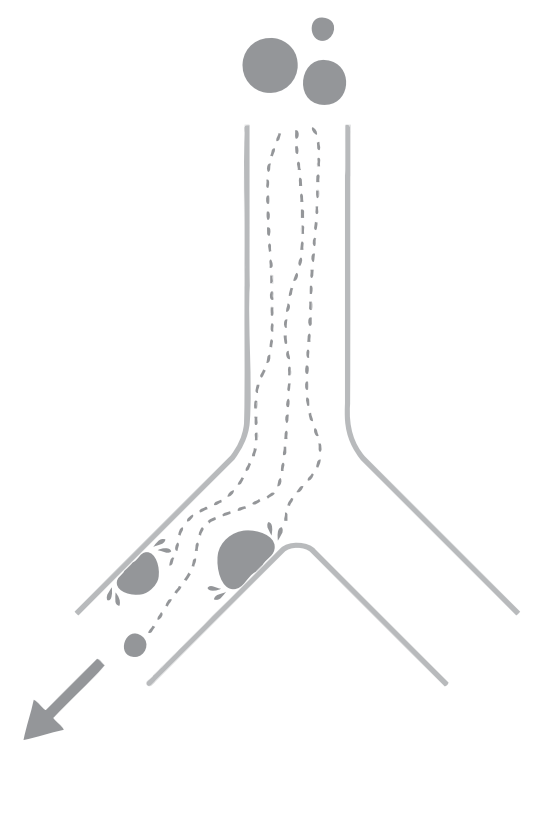

c)

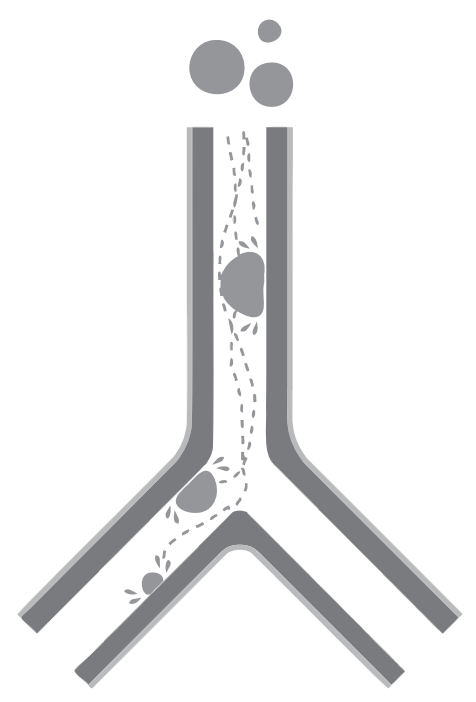

FIGURE 1 Schematic view of the bronchial tree showing the relation between airway size, flow velocity and deposition of three differently sized aerosol particles $(1,3$ and $5 \mu \mathrm{m})$. a) In the healthy lung, the 5 - $\mu \mathrm{m}$ particle has the highest probability of being deposited onto the mucosa of the central airways due to inertial impaction. b) In the healthy lung of a child, the airways are narrower and flow velocities of the inhaled particles are higher. As a result, the 3- and 5- $\mu \mathrm{m}$ particles are deposited on the central airway mucosa. c) In the diseased lung of a child, the airways are thickened due to mucosal swelling by inflammation and mucus. As a result, the cross-sectional diameter of the central airways is even smaller relative to the healthy lung (b); in addition, mucus depositions cause more turbulence of the inhaled air. As a result the 1-, 3- and 5- $\mu \mathrm{m}$ particles are deposited on the central airway mucosa. Reproduced and modified from [28] with permission from the publisher.

drug. In addition to the geometric size of the particle, the particle density determines transport velocity and deposition probability. Spheres that have the same transport velocity exhibit the same aerodynamic behaviour and have similar deposition patterns in the lung. This means that particles that are large geometrically and are porous (i.e. have a low density) will behave aerodynamically like particles that are small geometrically and are nonporous (i.e. have a high density). This effect of density on aerodynamic diameter is being used in the development of DPI drug formulations containing dry porous particles.

Patient-related determinants of lung deposition and distribution within the airways include, firstly, the diameter of the large airways. Children have smaller airways and higher inspiratory airflows relative to adults, both of which facilitate central airway deposition (fig. 1) [29]. The second patient-related factor determining particle deposition is the quality of the inhalation manoeuvre. This quality depends on age, physical capability, disease severity and the cognitive ability of the patient to perform specific inhalation manoeuvres. It is well recognised that even well-trained and capable patients might vary their inhalation technique considerably from day to day. A high inspiratory flow rate will result in more turbulence in the central airways and, therefore, result in an increased deposition of drug in the upper airways [30]. A slow inhalation manoeuvre, however, will result in less turbulence in central airways and, therefore, in a higher probability of aerosol particles bypassing the central large airways. Hence, ideally, an aerosol should be inhaled using a slow and deep inhalation so even large particles containing a high drug mass have a higher probability of bypassing the central large airways and making it all the way down into the diseased small airways. The third patient-related factor that determines particle deposition is the presence of structural abnormalities of the airways and/or mucus in the airways, which both can result in disturbance of the airflow pattern and thus in increased deposition at the sites of obstruction [30-32]. The fourth patientrelated factor is the ability of the lung to expand. Recent modelling studies showed that lobes with substantial structural damage received less inhaled antibiotic [32]. It is likely that structural abnormalities such as fibrosis in CF lungs have a negative impact on lung expansion (fig. 2) [33]. As a result, there is a preferential airflow to the healthier regions of the lung. 
a)

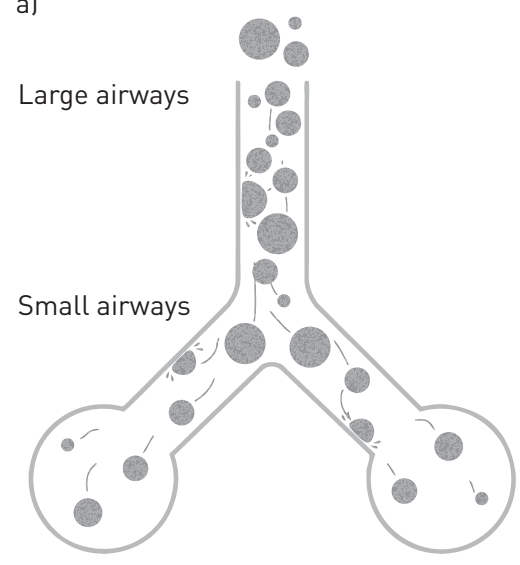

Parenchyma b)

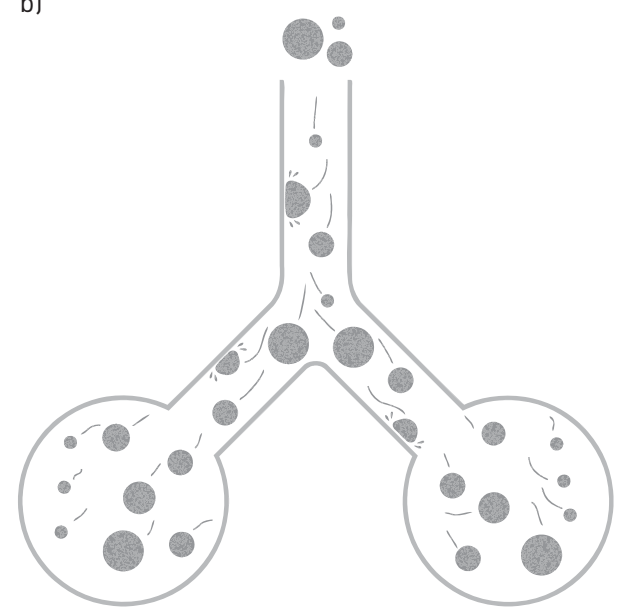

c)

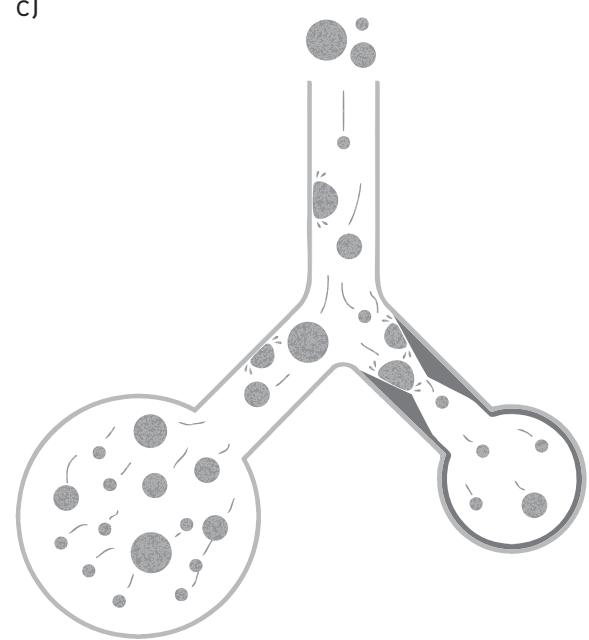

FIGURE 2 Schematic representation of the distribution of aerosol particles throughout the lung for the normal lung and the diseased lung. a) Distribution of aerosol particles for a patient with normal lung lobes while the patient is inhaling the antibiotic using tidal volume breathing. Note that there is homogeneous distribution of the antibiotic between lung segments, and deposition is higher in the central airways relative to the small airways and parenchyma. b) Same patient as in (a) inhaling deeply. Note that more drug reaches the small airways and parenchyma. Furthermore, there is equal expansion of the two lung lobes. c) The patient has considerable lung disease in one lobe, and is inhaling quickly and deeply. The diseased lobe has a higher airway resistance and reduced compliance relative to the healthier parts of the lung. The expansion of the diseased lobe is slower relative to the healthier parts. As a result, there is preferential flow to the healthier lobe. In addition, the partial obstruction of the airway to the diseased lung lobe causes a turbulent flow pattern and increased aerosol deposition. The overall result is that the healthy lung lobe receives more antibiotic relative to the diseased lobe.

Hence, to select the most appropriate inhalation device for a patient, we should not only take aerosol characteristics of the inhaled drug into account but also age, inhalation flow pattern related to the device and the severity of CF lung disease.

\section{Local concentrations of antibiotics}

It is generally believed that inhaled antibiotics are so effective because of the high sputum concentrations that were observed in the pivotal studies $[10,11]$. However, this concept is probably overly simplistic for a number of reasons. Firstly, the high concentrations measured in sputum are most likely to reflect drug primarily deposited in the large airways. As discussed, high central airway deposition can be the result of high turbulent flows leading to upper and central airway deposition, especially of larger inhaled particles, due to inertial impaction (fig. 3). Secondly, high concentrations in the central airways mean that less drug is available for the remainder of the bronchial tree, especially for the small airways. It should be kept in mind that drug bypassing the central airways is distributed over the vast surface area of the small airways. It is estimated that the total surface area of the small airways in adolescents is in the order of $1.2-1.3 \mathrm{~m}^{2}$. For each consecutive airway generation, concentrations will be lower as the total surface area of the airway surface increases exponentially. In addition, the velocity of airflow drops from central airways towards the small airways. This changes the principal mechanism of deposition by inertial impaction in more central large airways to sedimentation by gravitational forces in the smaller airways [34]. Thirdly, as discussed in the previous paragraph, diseased areas will receive a lower dose of the drug [32]. Downstream to the site of obstruction, less drug will be available for deposition. Fourthly, airflow is preferentially directed towards the healthier regions of the lung (fig. 2). Taking all these factors into account, it is highly likely that a wide range of sputum concentrations exist throughout the lung, and that concentrations in the small airways are likely to be low and might even fall below therapeutic levels depending of the mass of drug inhaled and on the aerosol characteristics [32]. Whether the use of a DPI or nebuliser for the inhalation of a specific antibiotic results in differences in the surface areas with very high and subinhibitory levels is unknown but should be considered.

What could the consequences of the aforementioned issues be for clinical practice? Firstly, it is unclear whether high antibiotic concentrations in central airways well above the minimum inhibitory concentration (MIC) translate to more effective killing of P. aeruginosa. If not, this should be considered a waste of drug that could better be delivered to the small airways and to more diseased regions with possibly subinhibitory concentrations. Secondly, low/subinhibitory concentrations in the more diseased and obstructed areas are not effective, and can lead to on-going infection in these regions. Hence, even when spirometry outcome measures improve during a cycle of inhaled antibiotic treatment, it is likely that the diseased areas of the lung remain undertreated. This could explain the effectiveness of treatment using intravenous rather than 

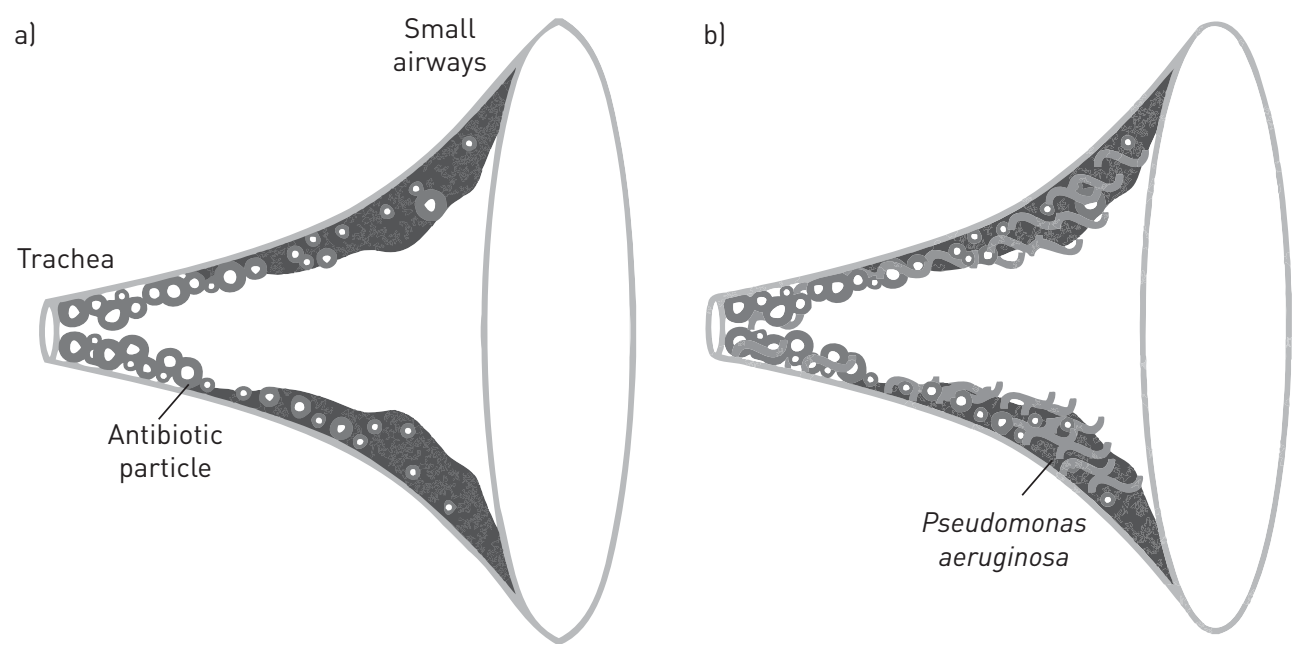

FIGURE 3 a) Schematic view of the total cross-sectional area of the bronchial tree. On the left, the narrow diameter of the trachea is shown. The total cross-sectional diameter and surface area of all small airways is substantially larger relative to the trachea. Note that the inflammatory thickening of the airway wall in the small airways is more severe in the small airways relative to the large airways. Furthermore, note that the density of deposited particles is larger and, thus, antibiotic concentration is higher in the central airways relative to the small airways. In addition, large and small particles are primarily deposited in the central large airway. In the small airways, more small particles can be observed and only few large particles. b) In the same bronchial tree as in (a), Pseudomonas aeruginosa are distributed throughout the bronchial tree, assuming equal density. Note that antibiotic concentration in the small airways is lower than in central airways; as a result, concentrations might fall below the minimal inhibitory concentration and be insufficient to adequately treat P. aeruginosa.

inhaled antibiotics, which may result in more effective antibiotic concentrations in diseased areas. Thirdly, subinhibitory concentrations can lead to the development of resistance. The development of resistance in patients on chronic treatment with inhaled antibiotics has been well studied. Indeed, for TIS, a small increase in resistance has been observed. However, this development of resistance did not correlate with any efficacy parameter [35], but it is doubtful that the resistance of expectorated sputum gives an accurate reflection of the distribution of resistance throughout the lung. It has been well recognised that the distribution of microorganisms in the CF lung is inhomogeneous. Hence, it is not surprising that, even for a single species, a wide distribution of MICs can exist [36, 37].

Clearly, for inhaled antibiotics, it is important to obtain sufficiently high concentrations throughout the bronchial tree. In the next sections, the basics and pro and cons of nebulisers and DPIs for the delivery of inhaled antibiotics are discussed for a better understanding of the relationship between the delivery device and airway concentrations of inhaled antibiotics.

\section{Wet nebulisers: the basics}

Nebulisers convert liquid medication into a mist and can be used to deliver a wide range of drug formulations for inhalation. There are different types of nebulisers, which have different mechanisms of aerosol generation. The differences in delivered aerosol between nebuliser systems currently available are significant [38]. There are jet, ultrasonic and vibrating mesh nebulisers, with or without smart nebuliser technology. The most frequently used systems to nebulise antibiotics are jet nebulisers. Jet nebulisers consist of a compressor that sucks air from the environment through an air filter and generates airflow through the nebuliser containing a Venturi tube. In the Venturi, the airflow is mixed with the fluid and a primary aerosol is formed. A baffle in the nebuliser further disintegrates the droplets into smaller aerosol particles. Most of the generated aerosol particles will fall back in the medication cup and will be re-nebulised. The patient inhales the aerosol while tidal breathing from a reservoir through a mouthpiece or face mask. For children, a good face mask design is important to maximise efficiency $[39,40]$. A child old enough to inhale through a mouthpiece should do so, as the efficiency of aerosol delivery can be doubled relative to inhalation by face mask [41].

The recently introduced mesh nebulisers use either a vibrating or fixed membrane with a piezoelectric element with microscopic holes to generate an aerosol. Vibrating mesh devices have a number of advantages over jet nebuliser systems. They are very efficient because there is almost no loss during exhalation and the residue after nebulisation is usually $<0.3 \mathrm{~mL}$, whereas in a jet nebuliser, $1-1.5 \mathrm{~mL}$ is left. Mesh nebulisers are silent and are generally portable, as they operate as effectively when using batteries as when using mains 
power. Lung deposition of mesh nebulisers is more efficient than conventional nebulisers, varying between $30 \%$ and $80 \%$ of the loading dose, depending on the device [42].

For smart nebulisers, the breathing pattern can be set in such a way that aerosol is only delivered during a pre-set fraction of the inspiration. In addition, for some nebulisers, the depth of the inhalation manoeuvre and the flow rate of the inhalation can be set. The deeper the inhalation, the shorter the nebulisation time. Almost no drug is lost during exhalation. Smart nebulisation systems can incorporate either jet or mesh nebulisers but the release of drug is controlled electronically, rather than allowing continuous release of drug. The I-neb (Philips Respironics, Parsippany, NJ, USA) is an example of such as system using a mesh nebuliser, while the Akita (Activaero, Munich, Germany) is an example of a smart system using a jet nebuliser.

Smart nebulisers are substantially more efficient and may achieve lung deposition of $60-80 \%$ of the loading dose (using mesh technology) [42], compared with 5-15\% with traditional jet nebulisers [43]. Furthermore, more efficient deposition of aerosol in the small airways can be achieved, which may result in more effective treatment of small airway obstruction [44].

Although there are many advantages, vibrating mesh and smart nebulisers are still not extensively tested in children and there is little clinical information available. Lung deposition is improved but evidence for dosage recommendations is either lacking or based on in vitro or adult data. New-generation nebulisers for inhaled antibiotics can be used as long as efficacy and toxicity data are available, especially when using potentially toxic antibiotics such as inhaled tobramycin.

\section{Nebulisers for inhaled antibiotics: pros and cons}

Inhaled antibiotics are registered for use with a specific nebuliser-compressor combination. Phase III studies with TIS were performed with the LC Plus nebuliser (Pari GmbH, Munich, Germany) with either the Pari Turbo Boy or PulmoAide compressor (DeVilbiss, Mannheim, Germany) [10]. The nebulisation of colistin is registered for use with the Pari LC Plus nebuliser and with the I-neb nebuliser in the UK. However, no proper phase III registration studies were conducted for nebulised colistin.

For the nebulisation of aztreonam for treatment of chronic $P$. aeruginosa airway infection, the Pari eFlow mesh nebuliser has been registered [16].

The use of nebulisers has some well-recognised advantages. 1) They are a platform to deliver drugs that are only available as fluids. 2) They can be used for all ages from infancy into adulthood. 3) The nebulised drug can be inhaled while the patient is breathing tidally. Hence, no specific inhalation manoeuvre is required. 4) Over the last few decades, smart nebulisers such as the I-neb and Akita have been developed that allow electronic data logging. This supplies objective information for patient and CF team on treatment adherence $[44,45]$. 5) Smart nebulisers such as the Akita allow the control of inhalation competence. Hence, the patient has to follow a pre-set inhalation profile, optimising treatment efficacy and efficiency. 6) Smart nebulisers can be set to efficiently target the small airways, which play an important role in CF lung disease $[3,46]$. To do so, a personalised inhalation profile can be programmed onto a smart card in the nebuliser. Improved delivery of dornase alfa to the small airways using the Akita nebuliser resulted in substantial improvement of small airways patency. Similar approaches could theoretically be of benefit to improve efficiency and efficacy of other nebulised drugs such as hypertonic saline and antibiotics.

There are some important disadvantages related to nebuliser therapy. 1) It is time-consuming. For patients on maintenance treatment with dornase alpha and inhaled antibiotics, nebuliser therapy can take up $2 \mathrm{~h}$ per day [47]. This time is needed for preparation of the nebuliser, nebulisation of the drug and cleaning the nebuliser after its use. For colistin, up to $10 \mathrm{~min}$ extra time is needed to dilute the appropriate dose in water for injection to obtain an isotonic solution. This is not the case for TIS or aztreonam lysine, which are readily available in a unit-dose vial. 2) The use of nebulisers includes the risk of contamination when not properly cleaned [48]. 3) Nebulisers are bulky and less portable than other devices. 4) Nebulisers require regular maintenance. Over time, the air filter of jet nebulisers gets polluted with dust particles and, thus, should be replaced at regular intervals. Similarly, "lifelong" nebulisers suffer from wear and tear, and thus require replacement once or twice a year. Furthermore, the compressor output of jet nebulisers should be periodically examined as per the manufacturer's instructions. For the Pari eFlow mesh nebuliser used for aztreonam for inhalation solution (AZLI; Cayston (Gilead Sciences Inc., Forest City, CA, USA)) therapy, maintenance issues are different. Occlusion of the holes can occur [49], which prolongs treatment time. Hence, they require careful cleaning after each use and frequent replacement of the mesh to prevent buildup of deposit and blockage of the apertures [49]. Therefore, a new mesh is delivered with each monthly package of aztreonam. In addition, the mesh should be replaced if nebulisation time exceeds 5 min. Overall, a great need was felt by the CF community and the pharmaceutical industry to develop more time-efficient, less cumbersome alternatives for nebulized antibiotic therapy. 


\section{DPIs: the basics}

In DPIs for antibiotics, the drug is present as a dry powder formulation in a capsule. The loading dose of the capsules for antibiotics is in the 20-150-mg range and, therefore, substantially higher than that of antiasthma drugs, which is mostly in the $50-200-\mu \mathrm{g}$ range. For this reason, multiple capsules can be needed to inhale a sufficient mass of the antibiotic into the lungs. The technical properties of the dry powder formulation combined with the properties of the inhaler determine how the powder can best be inhaled. Both the mass and the aerosol characteristics of the released aerosol depend on the inhaled volume and on the inspiratory flow profile generated by the patient.

TIP is formulated using PulmoSphere technology (Novartis AG, Basel, Switzerland). This is a spray-drying technique, which generates relatively large porous particles that disperse very easily. For each treatment, four capsules of $28 \mathrm{mg}$ TIP need to be inhaled through a low-resistance inhaler to get a lung dose equivalent to that of $300 \mathrm{mg}$ nebulised TIS. A low flow of $30 \mathrm{~L} \cdot \mathrm{min}^{-1}$ is sufficient to release the drug from the capsule and to disperse the aerosol particles. TIP has an MMAD $<4 \mu \mathrm{m}$ and a grain size distribution of 1.7-2.7 $\mu \mathrm{m}$ [50]. The low-resistance inhaler allows the patient to generate a wide range of inspiratory flows [51]. In a controlled laboratory setting, it was shown that almost all CF patients of 6 years and older were able to generate flows of $\geqslant 30 \mathrm{~L} \cdot \mathrm{min}^{-1}$. However, some patients obtained inspiratory flows as high as $170 \mathrm{~L} \cdot \mathrm{min}^{-1}$ through a low-resistance inhaler. Unfortunately, as discussed, high inspiratory flows increase oropharyngeal deposition and can reduce lung deposition, especially in the small airways. To empty all drug from the TIP capsule, an inspiratory volume of $1 \mathrm{~L}$ is sufficient to release all dry powder [51]. Most patients of 6 years and older were able to inhale a volume of $\geqslant 1 \mathrm{~L}$ [51]. However, to ensure that all drug is released from the capsule, it is recommended to repeat the inhalation manoeuvre twice for each capsule.

Colobreathe is formulated as micronised particles that, in general, do not disperse very easily. For each administration, one 125-mg capsule needs to be inhaled through a low-resistance inhaler to get a lung dose equivalent to that of $160 \mathrm{mg}$ nebulised colistin. It is claimed that an inspiratory flow of $30 \mathrm{~L} \cdot \mathrm{min}^{-1}$ through the inhaler is required to disperse the micronised drug optimally into respirable aerosol particles [16]. However, currently, there are no published data available describing the aerosol characteristics of the colistin DPI. To ensure that all drug is released from the capsule, it is recommended to repeat the inhalation manoeuvre twice for each capsule.

Clearly, the inspiratory flow and volume when inhaling an antibiotic from a DPI are important determinants of the deposition pattern and efficacy. Surprisingly, the optimal inhalation profiles of TIP and Colobreathe have not been clearly defined to date; this should be further investigated to optimise this form of inhaled antibiotic treatment. Next, patients should be trained to use the optimal inhalation technique with training aids and this technique should be regularly evaluated.

\section{DPIs: pros and cons}

DPIs have major advantages over nebulisers. 1) Administration is quick. For TIP, four capsules can be easily inhaled in 5 min. 2) DPIs do not require extensive cleaning after use. 3) Maintenance of the DPI is not required. 4) They are easy to carry around. 5) The capsules are packaged in sealed blisters and do not require refrigeration. Overall, this method of delivery is more convenient and sterile, which is an important consideration for CF patients who are highly susceptible to lung infections.

The most important disadvantage of a DPI inhaler is that the aerosol characteristics of the inhaled drug and, therefore, of the lung deposition can be highly dependent on the inhalation profile generated by the patient through the DPI. To our knowledge, no field studies have been published that observed how CF patients operate these DPIs in daily life. The DPIs for TIP and colistin are low-resistance inhalers. Hence, when a patient inhales forcefully, very high inspiratory flows can be obtained, resulting in a high oropharyngeal and central airway deposition [30]. In addition, this can result in cough. In the TIP versus TIS study, cough was reported as adverse event in $48 \%$ of the subjects on TIP versus $31 \%$ in the patients on TIS [19]. The optimal inhalation profile is formulation dependent. Hence, for the spray-dried, hollow porous particles of TIP, a slow and deep inhalation might be sufficient to disperse the drug while reducing upper airway deposition and improving deposition into the small airways. The optimal inhalation for the micronised colistin formulation is difficult to predict. It is likely that the dispersion of this formulation is highly flow dependent. A very high inspiratory flow might be needed to generate a sufficiently large fraction of small particles to treat the small airways effectively. For dry powder antibiotic formulations that are still in development, it will be important to educate prescribing physicians and patients to understand the key characteristics of each formulation (and device) and to be aware of the optimal inhalation profile required for that formulation. Another factor to consider is that when DPIs are prescribed to a patient for antibiotics and other medications that require different inhalation patterns (table 1), this is likely to result in confusion and erroneous use. Finally, we should investigate whether patients can be trained consistently "not to inhale 
TABLE 1 Inhaler devices for currently available inhaled medication in cystic fibrosis

\begin{tabular}{lccc} 
Inhaled drug & Nebuliser & DPI & pMDI \\
\hline Hypertonic saline (7\%) & + & - & - \\
Mannitol & - & + & - \\
Dornase alpha & + & - & - \\
Bronchodilators & + & + & + \\
Inhaled corticosteroids & + & + & + \\
Tobramycin & + & + & - \\
Colistin & + & + & - \\
Aztreonam & + & - & - \\
AmBisome & + & - & - \\
Liposomal amikacin & + & - & - \\
Ciprofloxacin & - & + & - \\
Vancomycin & - & + & - \\
Clarithromycin & - & + & - \\
\hline
\end{tabular}

AmBisome is manufactured by Gilead Sciences, Uxbridge, UK. DPI: dry powder inhaler; pMDI: pressurised, metered-dose inhaler.

too fast" or if the devices can be modified to ensure that patients inhale within the correct range of inspiratory flows (e.g. by increasing the device resistance or the use of visual/auditory aids).

\section{Dry or wet?}

Taking into account the pros and cons of nebulisers and DPIs for maintenance antibiotic treatment, it is clear that DPIs are more convenient for patients and less conspicuous to use, and from this perspective, the device of preference for patients. However, the most important reason for the physician to select one or the other should be primarily based on effectiveness. When selecting the potentially most effective inhalation device for/with the patient, several considerations should be taken into account (table 2). In the development programme of TIP, it was designed to be equally effective as TIS. To accomplish this, TIP was aimed to match the aerosol and pharmacokinetic characteristics of TIS as closely as possible. Hence, in phase I and II of the TIP development programme, a dose of TIP was selected to match the deposition characteristics and resulting pharmacokinetic profile of $300 \mathrm{mg}$ TIS. Eventually, four capsules each containing $28 \mathrm{mg}$ of the spray-dried tobramycin powder formulation equalled the pharmacokinetic profile of $300 \mathrm{mg}$ TIS. Next, the efficacy of the TIP formulation was tested against nebulised TIS in phase III trials including 553 patients in a noninferiority design with a $6 \%$ noninferiority margin using change in forced expiratory volume of $1 \mathrm{~s}$ (FEV1) \% predicted relative to baseline as the primary end-point. The response profile of TIP and TIS for FEV1 and colony-forming units were identical [10, 19]. However, despite the statistically proven noninferiority response pattern of TIP and TIS in the phase III regulatory trial, it is likely that differences in efficacy between TIP and TIS performance exist, taking into account the many differences

\section{TABLE 2 Considerations when prescribing inhaled antibiotics}

The concentration gradient of an inhaled antibiotic goes progressively down from central airways towards the small airways There is a preferential flow of inhaled antibiotic towards the more healthy regions of the lung

The more diseased the lung, the more inhomogeneous the deposition pattern and the more regions will be suboptimally treated Subinhibitory concentrations of inhaled antibiotics are likely to occur in advanced disease

Inhalation of an antibiotic by DPI is faster and cleaner relative to nebulised antibiotics

The aerosol characteristics of an inhaled antibiotic by DPI depend on formulation, device and inhalation manoeuvre

Each antibiotic inhaled by DPI has a device- and formulation-specific optimal inhalation profile

The patient (and parents in the case of children) should both be aware of the optimal inhalation profile

The efficacy of inhaled antibiotic therapy is determined by adherence and inhalation competence

Inhalation technique should be repeatedly evaluated and patients (parents) repeatedly trained

In case of a suboptimal therapeutic treatment result, check and recheck inhalation competence

For patients using a DPI but who cannot reproducibly generate the optimal inhalation profile, consider switching back to a nebuliser or to a smart nebuliser that guides the patient in optimising the inhalation manoeuvre

DPI: dry powder inhaler. 
between DPI and nebuliser platforms for delivery of tobramycin as discussed in the previous paragraphs. More subtle effects on efficacy might easily have remained unnoticed because of the noninferiority study design. For colistin, the comparison between the DPI and the nebuliser therapy is less clear as no comparator phase III trials have been conducted and the characteristics of the micronised dry powder formulation have not been described in detail in the literature. Better understanding of the impact of factors such as age, severity of disease and deposition variability on regular use of these devices are all of key importance in optimising the use of DPI antibiotic formulations.

For antibiotic treatment of newly acquired P. aeruginosa, nebulised antibiotics are considered the standard of treatment [9]. For patients without elevated $P$. aeruginosa antibodies, 1 month of nebulised TIS or AZLI has been shown to be effective in eradicating $P$. aeruginosa in up to $\sim 90 \%$ of patients $[7,15]$. However, positive $P$. aeruginosa antibodies or history of recurrent $P$. aeruginosa infection reduced the chance of successful eradication to $36-48 \%$ of patients. $P$. aeruginosa infection is associated with the development of structural lung abnormalities [52]. In the light of the issues discussed in previous paragraphs, it is quite possible that failure might be related to subinhibitory levels of inhaled antibiotics in areas of the lungs with structural disease. Taking into account the differences that can exist in the aerosol distribution pattern between TIS and TIP, it cannot automatically be assumed that TIP is equally effective as TIS in eradicating P. aeruginosa. Hence, until eradication studies with TIP or Colobreathe have been undertaken, the treatment options for first P. aeruginosa acquisition is TIS, AZLI or nebulised colistin [7, 15].

\section{Further optimisation}

The aforementioned issues offer considerable opportunities for further improvement of inhaled antibiotics. Current therapy is "one size fits all". Child or adult, early or advanced disease; are all treated with the same regimen. It might well be that for more advanced disease, a higher dose is needed to cover all airway generations with antibiotic concentrations above MIC. For TIP and TIS, a once-daily double dose might result in larger areas with concentrations above MIC and may therefore be more efficacious than current twice-daily administration. In addition, because of the difference in mechanism of action of various antibiotic classes, the frequency of administration should be optimised for each class. Advanced mathematical modelling can help us to determine this relationship and to design a specific dose relative to disease severity. Furthermore, it might be possible to improve the effectiveness of eradication therapy by increasing the dose in those patients with more advanced disease and in whom primary eradication therapy fails. Current DPI devices do not control the inhalation flow by the patient. Hence, there is an opportunity to optimise the inhalation manoeuvre of recently developed DPIs based on their characteristics. Training aids will be needed to facilitate this training. In the future, smart DPIs might be developed that guide the patient through the optimal inhalation manoeuvre.

\section{Conclusion}

Inhaled antibiotics are of key importance in the treatment of CF-related lung disease. Care should be taken to ensure that the small airways are efficiently targeted, even in diseased regions of the lung. Whether this is the case depends on many factors such as age, inhalation manoeuvre, severity of structural lung disease and other factors. Nebulisers are important especially for those inhaled antibiotics that are only available as a fluid. The use of nebulisers requires that technical maintenance is well organised. When possible, DPIs should be used to reduce the treatment burden. CF caregivers and patients should be made aware that there are major differences between the inhalation manoeuvre of a nebulised antibiotic and a DPI. Aerosol deposition by DPIs can vary widely in relation to the inhalation manoeuvre. Hence, switching a patient from a nebuliser to a DPI requires careful instruction of the optimal inhalation manoeuvre for that specific antibiotic. The optimal inhalation manoeuvre should be clearly defined by the pharmaceutical industry.

All aspects of inhaled antibiotic therapy should be carefully and frequently evaluated with the patient in the starting phase and, when used routinely, at least once a year. Alternative dose regimens for inhaled antibiotics need to be further investigated.

\section{References}

1 Sly PD, Brennan S, Gangell C, et al. Lung disease at diagnosis in infants with cystic fibrosis detected by newborn screening. Am J Respir Crit Care Med 2009; 180: 146-152.

2 Sly PD, Gangell CL, Chen L, et al. Risk factors for bronchiectasis in children with cystic fibrosis. N Engl J Med 2013; 368: 1963-1970.

3 Tiddens HAWM, Donaldson SH, Rosenfeld M, et al. Cystic fibrosis lung disease starts in the small airways: can we treat it more effectively? Pediatr Pulmonol 2010; 45: 107-117.

4 Loeve M, Hop WC, de Bruijne M, et al. Chest computed tomography scores are predictive of survival in patients with cystic fibrosis awaiting lung transplantation. Am J Respir Crit Care Med 2012; 185: 1096-1103.

5 Littlewood JM, Miller MG, Ghoneim AT, et al. Nebulised colomycin for early Pseudomonas colonisation in cystic fibrosis. Lancet 1985; 1: 865. 
Frederiksen B, Koch C, Hoiby N. Changing epidemiology of Pseudomonas aeruginosa infection in Danish cystic fibrosis patients (1974-1995). Pediatr Pulmonol 1999; 28: 159-166.

7 Ratjen F, Munck A, Kho P, et al. Treatment of early Pseudomonas aeruginosa infection in patients with cystic fibrosis: the ELITE trial. Thorax 2010; 65: 286-291.

8 Flume PA, O'Sullivan BP, Robinson KA, et al. Cystic fibrosis pulmonary guidelines: chronic medications for maintenance of lung health. Am J Respir Crit Care Med 2007; 176: 957-969.

9 Smyth AR, Bell SC, Bojcin S, et al. European Cystic Fibrosis Society standards of care: best practice guidelines. J Cyst Fibros 2014; 13: Suppl. 1, S23-S42.

10 Ramsey BW, Pepe MS, Quan JM, et al. Intermittent administration of inhaled tobramycin in patients with cystic fibrosis. N Engl J Med 1999; 340: 23-30.

11 Retsch-Bogart GZ, Quittner AL, Gibson RL, et al. Efficacy and safety of inhaled aztreonam lysine for airway pseudomonas in cystic fibrosis. Chest 2009; 135: 1223-1232.

12 Quittner AL, Buu A. Effects of tobramycin solution for inhalation on global ratings of quality of life in patients with cystic fibrosis and Pseudomonas aeruginosa infection. Pediatr Pulmonol 2002; 33: 269-276.

13 Ratjen F, Doring G, Nikolaizik WH. Effect of inhaled tobramycin on early Pseudomonas aeruginosa colonisation in patients with cystic fibrosis. Lancet 2001; 358: 983-984.

14 Gibson RL, Emerson J, McNamara S, et al. Significant microbiological effect of inhaled tobramycin in young children with cystic fibrosis. Am J Respir Crit Care Med 2003; 167: 841-9.

15 Tiddens HA, De Boeck K, Clancy JP, et al. Open label study of inhaled aztreonam for Pseudomonas eradication in children with cystic fibrosis: the ALPINE study. J Cyst Fibros 2014 [In press DOI: 10.1016/j.jcf.2014.06.003].

16 McCoy KS, Quittner AL, Oermann CM, et al. Inhaled aztreonam lysine for chronic airway Pseudomonas aeruginosa in cystic fibrosis. Am J Respir Crit Care Med 2008; 178: 921-928.

17 Jensen T, Pedersen SS, Garne S, et al. Colistin inhalation therapy in cystic fibrosis patients with chronic Pseudomonas aeruginosa lung infection. J Antimicrob Chemother 1987; 19: 831-8.

18 Conole D, Keating GM. Colistimethate sodium dry powder for inhalation: a review of its use in the treatment of chronic Pseudomonas aeruginosa infection in patients with cystic fibrosis. Drugs 2014; 74: $377-387$.

19 Konstan MW, Flume PA, Kappler M, et al. Safety, efficacy and convenience of tobramycin inhalation powder in cystic fibrosis patients: the EAGER trial. J Cyst Fibros 2011; 10: 54-61.

20 Schuster A, Haliburn C, Doring G, et al. Safety, efficacy and convenience of colistimethate sodium dry powder for inhalation (Colobreathe DPI) in patients with cystic fibrosis: a randomised study. Thorax 2013; 68: 344-350.

21 Wilson R, Welte T, Polverino E, et al. Ciprofloxacin dry powder for inhalation in non-cystic fibrosis bronchiectasis: a phase II randomised study. Eur Respir J 2013; 41: 1107-1115.

22 Stass H, Weimann B, Nagelschmitz J, et al. Tolerability and pharmacokinetic properties of ciprofloxacin dry powder for inhalation in patients with cystic fibrosis: a phase I, randomized, dose-escalation study. Clin Ther 2013; 35: 1571-1581.

23 Geller DE, Flume PA, Staab D, et al. Levofloxacin inhalation solution (MP-376) in patients with cystic fibrosis with Pseudomonas aeruginosa. Am J Respir Crit Care Med 2011; 183: 1510-1516.

24 Gaspar MC, Couet W, Olivier JC, et al. Pseudomonas aeruginosa infection in cystic fibrosis lung disease and new perspectives of treatment: a review. Eur J Clin Microbiol Infect Dis 2013; 32: 1231-1252.

25 Park CW, Li X, Vogt FG, et al. Advanced spray-dried design, physicochemical characterization, and aerosol dispersion performance of vancomycin and clarithromycin multifunctional controlled release particles for targeted respiratory delivery as dry powder inhalation aerosols. Int J Pharm 2013; 455: 374-392.

26 Rubin BK. Aerosolized antibiotics for non-cystic fibrosis bronchiectasis. J Aerosol Med Pulm Drug Deliv 2008; 21: 71-76.

27 Bilton D, Henig N, Morrissey B, et al. Addition of inhaled tobramycin to ciprofloxacin for acute exacerbations of Pseudomonas aeruginosa infection in adult bronchiectasis. Chest 2006; 130: 1503-10.

28 Tiddens HAWM. Facts and fiction in inhalation therapy. Ital J Pediatr 2003; 29: 39-43.

29 de Jongh FH, Rinkel MJ, Hoeijmakers HW. Aerosol deposition in the upper airways of a child. J Aerosol Med 2006; 19: 279-289.

30 Laube BL, Jashnani R, Dalby RN, et al. Targeting aerosol deposition in patients with cystic fibrosis: effects of alterations in particle size and inspiratory flow rate. Chest 2000; 118: 1069-1076.

31 Laube BL, Geller DE, Lin TC, et al. Positive expiratory pressure changes aerosol distribution in patients with cystic fibrosis. Respir Care 2005; 50: 1438-1444.

32 Bos AC, vos WG, De Backer JW, et al. Airways surface liquid consentrations of aztreonam lysine for inhalation in children with cystic fibrosis: a modelling study. J Cyst Fibros 2013; 12: Suppl. 1, S98.

33 Fouras A, Allison BJ, Kitchen MJ, et al. Altered lung motion is a sensitive indicator of regional lung disease. Ann Biomed Eng 2012; 40: 1160-1169.

34 Vinchurkar S, Backer LD, Vos W, et al. A case series on lung deposition analysis of inhaled medication using functional imaging based computational fluid dynamics in asthmatic patients: effect of upper airway morphology and comparison with in vivo data. Inhal Toxicol 2012; 24: 81-88.

35 Smith AL, Fiel SB, Mayer-Hamblett N, et al. Susceptibility testing of Pseudomonas aeruginosa isolates and clinical response to parenteral antibiotic administration: lack of association in cystic fibrosis. Chest 2003; 123: 1495-1502.

36 Perry JD, Laine L, Hughes S, et al. Recovery of antimicrobial-resistant Pseudomonas aeruginosa from sputa of cystic fibrosis patients by culture on selective media. I Antimicrob Chemother 2008; 61: 1057-1061.

37 Fothergill JL, Mowat E, Ledson MJ, et al. Fluctuations in phenotypes and genotypes within populations of Pseudomonas aeruginosa in the cystic fibrosis lung during pulmonary exacerbations. J Med Microbiol 2010; 59: 472-481.

38 Daniels T, Mills N, Whitaker P. Nebuliser systems for drug delivery in cystic fibrosis. Cochrane Database Syst Rev 2013; 4: CD007639.

39 Esposito-Festen JE, Ates B, van Vliet FJM, et al. Effect of a facemask leak on aerosol delivery from a pMDI-spacer system. I Aerosol Med 2004; 17: 1-6.

40 Smaldone GC, Sangwan S, Shah A. Facemask design, facial deposition, and delivered dose of nebulized aerosols. J Aerosol Med 2007; 20: Suppl. 1, S66-S75.

41 Chua HL, Collis GG, Newbury AM, et al. The influence of age on aerosol deposition in children with cystic fibrosis. Eur Respir J 1994; 7: 2185-2191. 
42 Nikander K, Prince I, Coughlin S, et al. Mode of breathing-tidal or slow and deep-through the I-neb Adaptive Aerosol Delivery (AAD) system affects lung deposition of ${ }^{99 \mathrm{~m}}$ Tc-DTPA. J Aerosol Med Pulm Drug Deliv 2010; 23 : Suppl. 1, S37-S43.

43 Laube BL, Janssens HM, de Jongh FH, et al. What the pulmonary specialist should know about the new inhalation therapies. Eur Respir J 2011; 37: 1308-1331.

44 Bakker EM, Volpi S, Salonini E, et al. Improved treatment response to dornase alfa in cystic fibrosis patients using controlled inhalation. Eur Respir J 2011; 38: 1328-35.

45 McNamara PS, McCormack P, McDonald AJ, et al. Open adherence monitoring using routine data download from an adaptive aerosol delivery nebuliser in children with cystic fibrosis. J Cyst Fibros 2009; 8: 258-263.

46 Bakker EM, Borsboom GJ, van der Wiel-Kooij EC, et al. Small airway involvement in cystic fibrosis lung disease: routine spirometry as an early and sensitive marker. Pediatr Pulmonol 2013; 48: 1081-1088.

47 Sawicki GS, Tiddens H. Managing treatment complexity in cystic fibrosis: challenges and opportunities. Pediatr Pulmonol 2012; 47: 523-533.

48 Blau H, Mussaffi $\mathrm{H}$, Mei Zahav M, et al. Microbial contamination of nebulizers in the home treatment of cystic fibrosis. Child Care Health Dev 2007; 33: 491-5.

49 Rottier BL, van Erp CJ, Sluyter TS, et al. Changes in performance of the Pari eFlow rapid and Pari LC Plus during 6 months use by CF patients. J Aerosol Med Pulm Drug Deliv 2009; 22: 263-269.

50 Geller DE, Weers J, Heuerding S. Development of an inhaled dry-powder formulation of tobramycin using PulmoSphere technology. J Aerosol Med Pulm Drug Deliv 2011; 24: 175-182.

51 Tiddens HAWM, Geller DE, Challoner P, et al. Effect of dry powder inhaler resistance on the inspiratory flow rates and volumes of cystic fibrosis patients of six years and older. J Aerosol Med 2006; 19: 456-465.

52 Robinson TE, Leung AN, Chen X, et al. Cystic fibrosis HRCT scores correlate strongly with Pseudomonas infection. Pediatr Pulmonol 2009; 44: 1107-1117. 